\title{
Detección fenotípica y genotípica de la producción de carbapenemasas tipo NDM-1 y KPC en enterobacterias aisladas en un laboratorio clínico en Maracay, Venezuela
}

\author{
Phenotypic and genotypic detection of the production of carbapenemases type NDM-1 and \\ KPC in isolated Enterobacteriaceae in a clinical laboratory in Maracay, Venezuela
}

\author{
Dayana Requena S. 1,2, Ysvette Vásquez C. 3,4, Alexander Gil T.1,2, Juruany Cedeño P.1, \\ María Chabin J. ${ }^{1}$, Eglhis Delgado R. ${ }^{1}$, Dorielys Díaz C. ${ }^{1}$ y Joselyn Dos Santos P. ${ }^{1}$
}

\begin{abstract}
${ }^{1}$ Instituto de Investigaciones Biomédicas "Dr. Francisco J. Triana-Alonso", Facultad de Ciencias de la Salud sede Aragua, Universidad de Carabobo, Maracay, Venezuela.

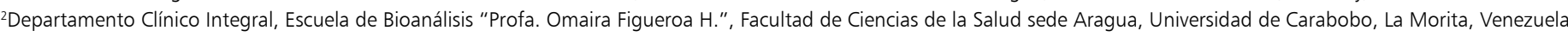
${ }^{3}$ Laboratorio Clínico Delgado Launois, C.A. Maracay estado Aragua, Venezuela.

${ }^{4}$ Servicio de Bacteriología, Hospital Estatal "Los Samanes", CORPOSALUD, Maracay, Venezuela.
\end{abstract}

Los autores declaran no tener conflictos de intereses.

La presente investigación no ha recibido ayudas específicas provenientes de agencias del sector público, sector comercial o entidades sin ánimo de lucro.

Recibido: 28 de julio de 2020 (segunda versión: 26 de enero de 2021) / Aceptado: 6 de febrero de 2021

\section{Resumen}

Introducción: La producción de $\beta$-lactamasas capaces de hidrolizar a los carbapenémicos es uno de los mecanismos de resistencia más preocupantes porque eliminan la última opción terapéutica frente a los microorganismos multi-resistentes. Objetivo: Determinar la producción de carbapenemasas tipo KPC y NDM-1, empleando métodos fenotípicos y genotípicos, en enterobacterias aisladas en un laboratorio clínico de la ciudad de Maracay, Venezuela. Métodos: Se determinó la producción de carbapenemasas mediante métodos fenotípicos (según algoritmo de Malbrán) y genotípicos (amplificación de los genes $b l a_{\mathrm{NDM}-1}$ y $b l a_{\mathrm{KPC}}$ por RPC) en enterobacterias aisladas en un laboratorio clínico durante el período marzo-agosto 2018. Resultados: Se identificaron 605 enterobacterias de diferentes especies, siendo Escherichia coli la cepa con mayor porcentaje de aislamiento $(61,3 \%)$, seguida por Klebsiella pneumoniae (14,9\%). Diez y seis enterobacterias $(2,64 \%)$ fueron positivas para la producción de carbapenemasas: 13 cepas de K. pneumoniae y tres del complejo Enterobacter cloacae. La RPC demostró que 14 cepas $(87,5 \%)$ contienen el gen $b l a_{\mathrm{NDM}-1} \mathrm{y}$ dos $(12,5 \%)$ el gen $b l a_{\mathrm{KPC}}$; se observó $100 \%$ de concordancia entre la determinación

\begin{abstract}
Background: The production of carbapenem-hydrolyzing $\beta$-lactamases is one of the most concerning resistance mechanisms since it eliminates the last therapeutic option against multidrug resistant microorganisms. Aim: To determine the production of KPC and NDM-1 type carbapenemases, using phenotypic and genotypic methods, in isolated enterobacteria in a clinical laboratory in the city of Maracay, Venezuela. Methods: The production of carbapenemases was determined by phenotypic (according to the Malbrán algorithm) and genotypic methods (amplification of the $b l a_{\mathrm{NDM}-1}$ and $b l a_{\mathrm{KPC}}$ genes by PCR) in clinical isolates of Enterobacteriaceae during the period March-August 2018. Results: 605 Enterobacteriaceae of different species were identified, being Escherichia coli the strain with the highest percentage of isolation $(61.3 \%)$, followed by Klebsiella pneumoniae (14.9\%). Sixteen strains $(2.64 \%)$ were positive for carbapenemases production: 13 strains of $K$. pneumoniae and three of the Enterobacter cloacae complex. PCR showed that 14 strains $(87.5 \%)$ carry the $b l a_{\mathrm{NDM}-1}$ gene and two strains $(12.5 \%)$ the $b l a_{\mathrm{KPC}}$ gene; $100 \%$ agreement was observed between phenotypic determination and PCR for both groups
\end{abstract}

\footnotetext{
Correspondencia a:

Dayana Requena Sarcolira

dayanacrs@gmail.com
} 
fenotípica y la RPC para ambos grupos de enzimas. Conclusiones: Los resultados mostraron mayor incidencia de la metalo- $\beta$-lactamasa tipo NDM-1, reconocida como una alarma epidemiológica debido a que su rápida diseminación dificulta su control, por lo que la identificación del tipo de enzima permitiría establecer estrategias de manejo y control más certeras con la finalidad de erradicar a dichos patógenos.

Palabras clave: enterobacterias; resistencia bacteriana; carbapenemasas; NDM-1, KPC.

\section{Introducción}

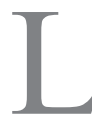

as enterobacterias son patógenos humanos comunes y colonizadores del tracto intestinal humano que pueden causar una amplia gama de enfermedades, incluyendo infecciones del tracto urinario, neumonía, infecciones del torrente sanguíneo, intra-abdominales, de piel y tejidos blandos en entornos comunitarios y hospitalarios ${ }^{1,2}$.

La resistencia a antimicrobianos es considerada un fenómeno evolutivo natural, que han desarrollado las bacterias como mecanismo de defensa; ante esto, cuentan con la producción de enzimas inactivadoras, mutación de sitios de acción, bombas de expulsión, entre otros $^{3}$. En los últimos años se ha generado alarma y preocupación por la gran dispersión de los bacilos gramnegativos resistentes a los carbapenémicos en los que el mecanismo implicado es la producción de $\beta$-lactamasas capaces de hidrolizar este grupo de antimicrobianos y que se han asociado a elementos genéticos transferibles. Entre las de mayor interés se encuentran las $\beta$-lactamasas de espectro extendido (BLEE) y las carbapenemasas ${ }^{4}$.

Las carbapenemasas, son enzimas inactivadoras de carbapenémicos, antimicrobianos $\beta$-lactámicos de amplio espectro con actividad bactericida frente a bacterias grampositivas y gramnegativas, tanto aeróbicas como anaeróbicas. Son utilizados sólo en cuadros de gran resistencia bacteriana, por lo que, este mecanismo de resistencia inhibe prácticamente el último escalón terapéutico frente a microorganismos gramnegativos multi-resistentes ${ }^{3}$.

Se clasifican en el sistema de Ambler de la siguiente manera: las carbapenemasas de clase A (GES, KPC) que son inhibidas por el ácido clavulánico; las de clase B o metalo- $\beta$-lactamasas (VIM, IMP, NDM) que son inhibidas por el ácido etilen-diamino-tetra-acético (EDTA); y las oxacilinasas de clase D que no se ven afectadas por el ácido clavulánico o el EDTA ${ }^{5}$.

Dentro de las carbapenemasas de clase A, las que mayor importancia epidemiológica tienen son las denominadas KPC que reciben este nombre por haberse encontrado inicialmente en Klebsiella pneumoniae $(\mathrm{KPC}=\text { K. pneumoniae carbapenemases })^{6,7}$. Por el momento se conocen 61 variantes (gen $b l a_{\mathrm{KPC}-61}$, GenBank MK559426.1), siendo KPC-2 y KPC-3 las variantes descritas con mayor frecuencia en Latinoamérica ${ }^{3}$.

El grupo más importante de carbapenemasas lo constituyen las metalo- $\beta$-lactamasas (MBL) pertenecientes a la clase $B$. La más recientemente descrita es la enzima Nueva Delhi metalo- $\beta$-lactamasa (NDM) que ha creado una importante alarma mediática debido al perfil muti-resistente o pan-resistente de los aislados que la producen. Esta of enzymes. Conclusions: The results of this study showed a higher incidence of metallo- $\beta$-lactamase type NDM-1, which rapid dissemination and consequently difficult control has been cause of epidemiological alert. The identification of the type of enzyme would allow establishing more accurate management and control strategies in order to eradicate these pathogens.

Keywords: Enterobacteriaceae; resistance mechanisms; carbapenemase; NDM-1, KPC. enzima, cuyo origen podría situarse en la India, se detectó por primera vez en K. pneumoniae y también está presente en Escherichia coli y en otras enterobacterias, y asimismo en Acinetobacter spp. ${ }^{8,9}$.

En un estudio realizado en Venezuela, se evidenció la variabilidad de aislados productores de carbapenemasa de tipo KPC, describiendo aislados que permanecieron en el tiempo y la posible diseminación inter e intrahospitalaria en aislados que se encontraron genéticamente relacionados ${ }^{10}$. Sin embargo, son pocos los estudios sobre la existencia de otros tipos de carbapenemasas como la NDM-1. Por esa razón, el objetivo de este estudio fue determinar la producción de carbapenemasas tipo KPC y NDM-1, empleando métodos fenotípicos y genotípicos, en enterobacterias aisladas en un laboratorio clínico de la ciudad de Maracay, estado Aragua.

\section{Material y Métodos}

Se realizó la determinación de carbapenemasas a las enterobacterias aisladas de los cultivos procesados en el Servicio de Bacteriología de un laboratorio clínico, ubicado en Maracay, estado Aragua, Venezuela, durante el período marzo-agosto de 2018 , sin importar el tipo de muestra, edad o sexo del paciente.

Cada muestra se inoculó de manera manual en los distintos medios de cultivo a utilizar. Se observó el desarrollo bacteriano en las placas de agar MacConkey como colonias rojas que corresponden a bacterias que han metabolizado la lactosa debido al indicador de $\mathrm{pH}$ ácido (rojo neutro). La identificación de enterobacterias, se realizó mediante el uso de pruebas bioquímicas convencionales siguiendo el protocolo estándar de las bacterias gramnegativas. Una vez realizada la identificación del microorganismo se evaluó la susceptibilidad a los diferentes antimicrobianos (placa estratégica) siguiendo las recomendaciones del CLSI $2018^{11}$ (por sus siglas en inglés Clinical and Laboratory Standards Institute), empleando el método de difusión en disco por la técnica de Kirby-Bauer ${ }^{12}$.

\section{Detección fenotípica de carbapenemasas}

Se utilizó el algoritmo de trabajo establecido por el Instituto Nacional de Enfermedades Infecciosas "Dr. Carlos Malbrán" de Argentina, consensuado para la Red Latinoamericana de Vigilancia de la Resistencia Antimicrobiana (RELAVRA), el cual indica que el primer paso para la identificación del mecanismo de resistencia tipo KPC o MBL, es evaluar la disminución de la susceptibilidad antimicrobiana 
de la siguiente manera: 371 cepas de E. coli $(61,3 \%), 90$ cepas de K. pneumoniae (14,9\%), 74 cepas de Proteus spp. $(12,2 \%)$, y otras especies en menor proporción (Tabla 2).

\section{Detección fenotípica de carbapenemasas}

Se realizó el antibiograma a las 605 cepas de enterobacterias, obteniéndose como resultado que 16 cepas $(2,64 \%)$ presentaron un patrón de multi-resistencia sugestivo de carbapenemasas positivas (halo de inhibición $<22 \mathrm{~mm}$ para el disco de IPM), siendo 13 aislados de la especie K. pneumoniae $(81,25 \%)$ y tres se identificaron como complejo Enterobacter cloacae (18,75\%). Estas cepas fueron aisladas de forma independiente a partir de muestras de pacientes internados en diferentes centros hospitalarios de la ciudad de Maracay, estado Aragua, Venezuela. En ningún caso se aisló más de una cepa por paciente. El origen de las muestras fue: secreciones (n: 6), hemocultivos (n: 4), punta de catéter (n: 3), y en menor proporción orina (n: 1), líquido cefalorraquídeo (n: 1) y exudado uretral (n: 1).

\begin{tabular}{|ccc|}
\hline \multicolumn{2}{|c|}{ Tabla 1. Cebadores empleados en el estudio } & \\
\hline Nombre & \multicolumn{1}{c|}{ Secuencia } & Tamaño del producto \\
NDM-1 directo & 5'-GGTTTGGCGATCTGGTTTTC-3' $^{\prime}$ & $621 \mathrm{pb}$ \\
NDM-1 reverso & 3'-CGGAATGGCTCATCACGATC-5' $^{\prime}$ & \\
KPC directo & 5'-CGTCTAGTTCTGCTGTCTTG-3' $^{\prime}$ & $798 \mathrm{pb}$ \\
KPC reverso & 3'-CTTGTCATCCTTGTTAGGCG-5' $^{\prime}$ & \\
\hline Poirel y cols. 2011'19. & & \\
\hline
\end{tabular}

primer paso fue una desnaturalización inicial a $95^{\circ} \mathrm{C}$ por 5 min, seguida de 34 ciclos de desnaturalización ( 1 min a $\left.95^{\circ} \mathrm{C}\right)$, hibridación por $1 \mathrm{~min}\left(52^{\circ} \mathrm{C}\right.$ para NDM-1 y $55^{\circ} \mathrm{C}$ para $\mathrm{KPC}$ ), y extensión a $72^{\circ} \mathrm{C}$ por $1 \mathrm{~min}$, con un último ciclo de desnaturalización a $95{ }^{\circ} \mathrm{C}$ por $1 \mathrm{~min}$, extensión final por 5 min a $72{ }^{\circ} \mathrm{C}$ y enfriamiento a $4^{\circ} \mathrm{C}$ por $10 \mathrm{~min}$.

Los productos de amplificación se analizaron mediante electroforesis en gel de agarosa al 1,5\% en tampón TBE (Tris 90 mM, ácido bórico 90 mM, EDTA 2 mM, pH 8,3). Las imágenes de los patrones electroforéticos obtenidas por trans-iluminación UV, se digitalizaron en el equipo GelDoc 1000 (BIO-RAD) y se analizaron con el programa Quantity One (BIO-RAD).

Las reacciones de amplificación para los genes se consideraron positivas al observar una banda única de $621 \mathrm{pb}$ para el gen $b l a_{\mathrm{NDM}-1}$ y de $798 \mathrm{pb}$ para el gen $b l a_{\mathrm{KPC}}$.

\section{Resultados}

Para el estudio se incluyó un total de 2.671 muestras, de las cuales se identificaron 605 enterobacterias de diferentes géneros y especies, que estuvieron distribuidas

\begin{tabular}{|c|c|c|c|c|c|c|}
\hline Estudio & $\begin{array}{l}\text { Copro- } \\
\text { cultivo }\end{array}$ & $\begin{array}{c}\text { Secreción } \\
\text { o líquido } \\
\text { estéril }\end{array}$ & $\begin{array}{l}\text { Hemo- } \\
\text { cultivo }\end{array}$ & $\begin{array}{l}\text { Uro- } \\
\text { cultivo }\end{array}$ & Total & $\%$ \\
\hline BGN sin identificación & 0 & 0 & 1 & 0 & 1 & 0,2 \\
\hline Escherichia coli & 2 & 73 & 0 & 296 & 371 & 61,3 \\
\hline Complejo Enterobacter cloacae & 0 & 12 & 2 & 0 & 14 & 2,3 \\
\hline Enterobacter spp. & 0 & 5 & 7 & 6 & 18 & 3,0 \\
\hline Klebsiella pneumoniae & 0 & 37 & 6 & 47 & 90 & 14,9 \\
\hline Klebsiella spp. & 0 & 4 & 1 & 4 & 9 & 1,5 \\
\hline Pantoea agglomerans & 0 & 1 & 0 & 0 & 1 & 0,2 \\
\hline Proteus spp. & 0 & 43 & 0 & 31 & 74 & 12,2 \\
\hline Providencia spp. & 0 & 8 & 0 & 0 & 8 & 1,3 \\
\hline Serratia marcescens & 0 & 5 & 13 & 1 & 19 & 3,1 \\
\hline Total & 2 & 188 & 30 & 385 & 605 & 100 \\
\hline
\end{tabular}


Con las pruebas fenotípicas Blue-Carba y THT se obtuvo un resultado positivo para carbapenemasas en $100 \%$ de las cepas sospechosas. Adicionalmente, en $87,5 \%$ de los aislados se evidenció la sinergia entre los discos de EDTA y los carbapenémicos evaluados, demostrando la producción MBL; 12 cepas corresponden a la especie $K$. pneumoniae y dos del complejo E. cloacae. El 12,5\% de los aislados mostró sinergia entre los discos de APB y los carbapenémicos utilizados, demostrando la producción de serinoenzimas. Este resultado se reprodujo al realizar la prueba confirmatoria de carbapenemasas, e indican que, en las muestras analizadas en este estudio, existe una mayor prevalencia de carbapenemasas tipo MBL en comparación con el tipo KPC.

En la Tabla 3 se muestran los resultados de las pruebas fenotípicas realizadas para la detección de carbapene- masas en las 16 muestras que presentaron un halo de inhibición $<22 \mathrm{~mm}$ para el disco de IPM. Todas las técnicas mostraron $100 \%$ de sensibilidad.

\section{Detección de genes de resistencia}

En la amplificación de genes que codifican carbapenemasas, los ensayos de RPC mostraron que en los aislados clínicos positivos fenotípicamente para la producción de carbapenemasas, 87,5\% (n: 14) tienen el gen $b l a_{\mathrm{NDM}-1}$ y $12,5 \%$ (n: 2) son portadores del gen $b l a_{\mathrm{KPC}}$. En ambos ensayos de RPC, no se observó la amplificación de productos secundarios inespecíficos (Figura 1). Estos resultados concuerdan con la identificación fenotípica realizada con la prueba de sinergia con discos de EDTA y APB y con la prueba confirmatoria de carbapenemasas.

Tabla 3. Detección fenotípica de carbapenemasas según el algoritmo de Malbrán

\begin{tabular}{|c|c|c|c|c|c|}
\hline & \multicolumn{5}{|c|}{ Resultados positivos según el método fenotípico ensayado } \\
\hline & \multirow[t]{2}{*}{ Blue-Carba } & \multicolumn{2}{|c|}{ Sinergia con doble disco } & \multirow[t]{2}{*}{ Hodge modificado } & \multirow{2}{*}{$\begin{array}{l}\text { Prueba confirmatoria } \\
\text { (MASTDISCS }{ }^{\text {TM }} \text { Combi) }\end{array}$} \\
\hline & & EDTA/IPM & APB/IPM & & \\
\hline$n=16$ & $16 / 16$ & $14 / 16$ & $2 / 16$ & $16 / 16$ & $16 / 16$ \\
\hline Sensibilidad (\%) & 100 & 100 & 100 & 100 & 100 \\
\hline
\end{tabular}

A

$\begin{array}{lllllllllll}1 & 2 & 3 & 4 & 5 & 6 & 7 & 8 & 9 & 10 & 11\end{array}$

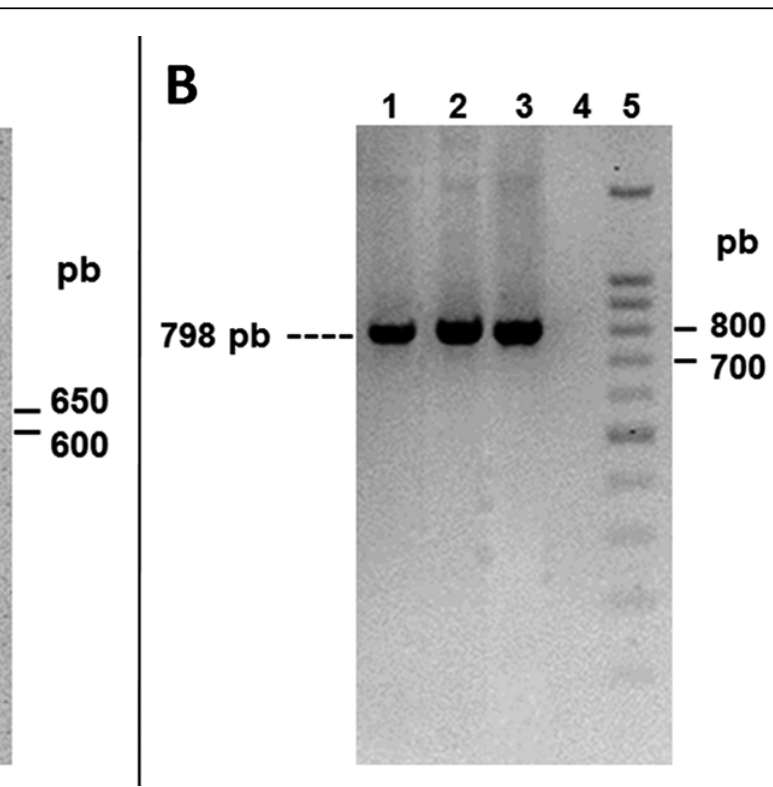

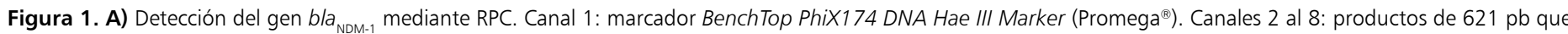

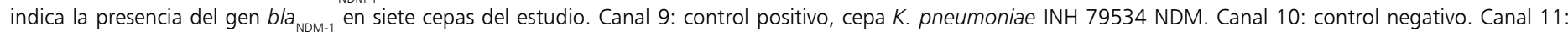

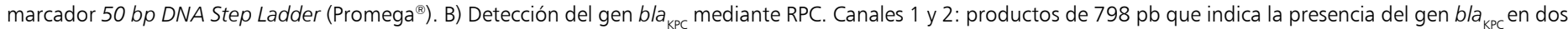

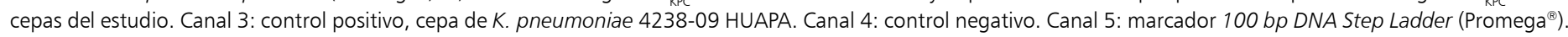


2016, en dos cepas de E. coli aisladas de pacientes en un centro hospitalario ${ }^{27}$. En cuanto al tipo KPC, un estudio publicado en el año 2019 mostró la amplia distribución del gen $b l a_{\text {KPC }}$ en diferentes estados de Venezuela ${ }^{10} \mathrm{y}$, recientemente, Martínez y cols., ${ }^{28}$ identificaron cuatro cepas de K. pneumoniae productoras de carbapenemasas por métodos fenotípicos, y la RCP demostró la co-existencia de los genes $b l a_{\mathrm{NDM}}$ y $b l a_{\mathrm{KPC}}$ en las cuatro cepas.

En Latinoamérica, $K$. pneumoniae es la principal productora de KPC y NDM- ${ }^{29}$ y se ha demostrado que es la primera en causar la transmisión del gen $b l a_{\mathrm{NDM}-1}$ a otras enterobacterias como las del complejo E. cloacae, siendo la segunda especie resistente a carbapenems aislada en este estudio. Si bien la presencia de carbapenemasas es poco frecuente en el complejo E. cloacae, en la última década se han descrito carbapenemasas de clase A (en especial KPC), clase B y clase D (en especial OXA-48) con frecuencias variables entre países, lo que ha contribuido al aumento de la resistencia a carbapenémicos y dificultado su manejo ${ }^{30}$. En este trabajo se observó mayor aislamiento de $b l a_{\mathrm{NDM}-1}(66,7 \%)$ en comparación con $b l a_{\mathrm{KPC}}(33,3 \%)$ en el complejo E. cloacae. Estos resultados son similares a los observados en un estudio previo donde, se reportó una alta incidencia $(72,7 \%)$ y propagación endémica de aislados de E. cloacae resistentes a carbapenem, productores de la enzima tipo NDM- $1^{31}$, lo que indica la diseminación de este gen hacia especies diferentes a $K$. pneumoniae.

El hecho de que en este estudio se encontró mayor frecuencia del gen $b l a_{\mathrm{NDM}-1}$ en comparación con el gen bla $_{\mathrm{KPC}}$, tanto en $K$. pneumoniae como en el complejo E. cloacae, resulta preocupante, ya que NDM-1 ha sido reconocido como una alarma epidemiológica y, por lo tanto, un importante tema de salud pública debido a que su rápida diseminación dificulta su control en las enfermedades infecciosas ${ }^{32}$. Estudios sugieren que parece haber una posible transferencia de $b l a_{\mathrm{NDM}-1}$ in vivo desde $K$. pneumoniae a $E$. coli o viceversa ${ }^{33}$. Esto se explica porque NDM-1 puede encontrarse en plásmidos conjugativos ${ }^{32}$. Este tipo de plásmidos tiene la capacidad de promover la diseminación horizontal de un gran número de genes de resistencia, contribuyendo al incremento de las poblaciones bacterianas resistentes y promoviendo la aparición de cepas patógenas multi-resistentes ${ }^{34}$. De esta manera, poseen una amplia variedad de hospederos e incluso garantiza su fácil transferencia entre géneros de la familia Enterobacteriaceae, así como en Pseudomonas spp. ${ }^{32}$.

Teniendo en cuenta que los carbapenem son el último recurso para tratar infecciones graves causadas por Enterobacteriaceae, es obligatorio controlar la diseminación de los mecanismos de resistencia a estos antimicrobianos ${ }^{3}$. La producción de carbapenemasas puede ser identificada a través de pruebas fenotípicas implementadas en los laboratorios de microbiología. Debido a la versatilidad de 
estas enzimas, hasta la fecha, no existe una sola prueba fenotípica con la suficiente capacidad de detección y diferenciación de todos los tipos de enzimas que existen. En la actualidad, el uso de métodos moleculares basados en la RPC, constituye la técnica más confiable de detección de este tipo de enzimas. La elevada especificidad y sensibilidad, y el poco tiempo requerido, hacen de las técnicas moleculares las más apropiadas ${ }^{35,36}$.

Sin embargo, los resultados obtenidos en el presente trabajo demuestran que el empleo de diferentes métodos fenotípicos permite sugerir el tipo de enzima presente en el microorganismo, ya que se observó 100\% de concordancia en los resultados obtenidos por esta metodología en comparación con la RPC, lo que adquiere gran importancia, sobre todo en los laboratorios con recursos limitados, que no cuentan con los reactivos y equipos necesarios para la aplicación de técnicas moleculares que permitan la detección del gen específico que produce la carbapenemasa.

Una de las limitantes del trabajo es no poder realizar la relación genética entre los aislados mediante electroforesis de campo pulsado o por ERIC-PCR (en inglés enterobacterial repetitive intergenic consensus sequence-based $P C R$ ), por lo que en este momento no es posible determinar cuáles son los clones que circulan en el estado Aragua. La rápida detección de bacterias productoras de carbapenemasas y la diferenciación del tipo de enzima presente, es de gran importancia para estudios epidemiológicos que permitirían tomar medidas de control con el fin de evitar la diseminación de estas bacterias a nivel hospitalario.

\section{Conclusiones}

El presente estudio muestra que $2,64 \%$ de las enterobacterias aisladas en el período establecido son resistentes a carbapenémicos y de éstas, $87,5 \%$ son portadoras del gen $b l a_{\mathrm{NDM}-1}, \mathrm{y} 12,5 \%$ poseen el gen $b l a_{\mathrm{KPC}}$. Las especies identificadas como portadoras de estos genes fueron $K$. pneumoniae y complejo E. cloacae. Este estudio representa el primer reporte de enterobacterias productoras de carbapenemasas tipo NDM-1 en el estado Aragua, Venezuela.

Agradecimientos. Al Laboratorio Clínico Delgado Launois, por permitirnos realizar parte del trabajo en sus instalaciones, y al Dr. Marcos De Donato de la Escuela de Ingeniería y Ciencias del Tecnológico de Monterrey, por facilitarnos los cebadores para amplificar el gen $b l a_{\mathrm{KPC}}$ y los ADN utilizados como control en este trabajo.

\section{Referencias bibliográficas}

1.- Wang J, Wu U, Lauderdale T, Chen M, Li $\mathrm{S}, \mathrm{Hsu} \mathrm{L}$, et al. Carbapenem-nonsusceptible Enterobacteriaceae in Taiwan. PLoS One 2015; 10:e0121668. doi: 10.1371/journal. pone. 0121668 .

2.- Hammoudi D, Ayoub Moubareck C, Aires J, Adaime A, Barakat A, Fayad N, et al. Countrywide spread of OXA-48 carbapenemase in Lebanon: surveillance and genetic characterization of carbapenem-non-susceptible Enterobacteriaceae in 10 hospitals over a oneyear period. Int J Infect Dis. 2014; 29: 139-44. doi: 10.1016/j.ijid.2014.07.017.

3.- García M. Carbapenemasas, una amenaza actual. Rev Cub Med Int Emerg. 2012; 11 (4): 2613-8. https://www.medigraphic.com/pdfs/ revcubmedinteme/cie-2012/cie124e.pdf

4.- Navarro F, Calvo J, Cantón R, FernándezCuenca F, Mirelis B. Detección fenotípica de mecanismos de resistencia en microorganismos gramnegativos. Enferm Infecc Microbiol Clin. 2011; 29-(7): 524-34. doi: 10.1016/j. eimc.2011.03.011.

5.- Sahin K, Tekin A, Ozdas S, Akin D, Yapislar H, Dilek A, et al. Evaluation of carbapenem resistance using phenotypic and genotypic techniques in Enterobacteriaceae isolates.
Ann Clin Microbiol Antimicrob. 2015; 14: 44 doi: 10.1186/s12941-015-0105-1.

6.- Nordmann P, Cuzon G, Naas T. The real threat of Klebsiella pneumoniae carbapenemaseproducing bacteria. Lancet Infect Dis. 2009; 9: 228-36. doi: 10.1016/S1473-3099(09)70054-4.

7.- Curiao T, Morosini M, Ruíz-Garbajosa P, Robustillo A, Baquero F, Coque T, et al. Emergence of bla $a_{\mathrm{KPC}-3}-\mathrm{Tn} 4401 \mathrm{a}$ associated with a pKPN3/4-like plasmid within ST384 and ST388 Klebsiella pneumoniae clones in Spain J Antimicrob Chemother. 2010; 65: 1608-14 doi: $10.1093 / \mathrm{jac} / \mathrm{dkq} 174$

8.- Walsh T R. Emerging carbapenemases: a global perspective. Int J Antimicrob Agents 2010; 36: S8-14. doi: 10.1016/S0924-8579(10)70004-2.

9.- Kumarasamy K, Toleman M, Walsh T, Bagaria J, Butt F, Balakrishnan R, et al. Emergence of a new antibiotic resistance mechanism in India, Pakistan, and the UK: a molecular, biological, and epidemiological study. Lancet Infect Dis. 2010; 10:5 97-602. doi: 10.1016/S14733099(10)70143-2.

10.- Cuaical-Ramos N M, Montiel M, Marcano Zamor D. Variabilidad genética de Klebsiella pneumoniae con carbapenemasa tipo KPC proveniente de diferentes estados de Venezuela. Enferm Infecc Microbiol Clin. 2019; 37 (2): 76-81. doi: 10.1016/j.eimc.2017.12.004.
11.- Clinical and Laboratory Standards Institute (CLSI). Performance Standards for Antimicrobial Susceptibility Testing: Twentyeighth Informational Supplement M100-S28, Wayne, PA (USA), 2018.

12.- Bauer A W, Kirby W M, Sherris J C, Turck M. Antibiotic susceptibility testing by a standardized single disk method. Am J Clin Pathol. 1966; 45 (4): 493-6. PMID: 5908210

13.- Servicio Antimicrobianos, Instituto Nacional de Enfermedades Infecciosas, Administración Nacional de Laboratorios e Institutos de Salud "Dr. Carlos G. Malbrán". Algoritmos 2016 Enterobacterias [Internet]. Buenos Aires: INEI, ANLIS; 2016. Disponible en: http://antimicrobianos.com.ar/ATB/wpcontent/uploads/2014/10/Algoritmos-2016Enterobacterias1.pdf.

14.- Marcano D, De Jesús A, Hernández L, Torres L. Frecuencia de enzimas asociadas a sensibilidad disminuida a betalactámicos en aislados de enterobacterias, Caracas, Venezuela. Rev Panam Salud Pública 2011; 30 (6): 529-34. PMID: 22358397.

15.- Velásquez J, Hernández R, Pamo O, Candiotti M, Pinedo Y, Sacsaquispe R, et al. Klebsiella pneumoniae resistente a los carbapenemes. Primer caso de carbapenemasa tipo KPC en Perú. Rev Soc Perú Med Interna 2013; 26 (4): 
192-6. http://revistamedicinainterna.net/index. php/spmi/article/view/214.

16.- Pires A, Novais L. Blue-Carba, an easy biochemical test for detection of device carbapenemase producers directly from bacterial cultures. J Clin Microbiol. 2013; 51 (12): 4281-3. doi: 10.1128/JCM.01634-13.

17.- Pasteran F, González L, Albornoz E, Bahr G, Vila A, Corso A. Triton Hodge test: improved protocol for modified Hodge test for enhanced detection of NDM and other carbapenemase producers. J Clin Microbiol. 2016; 54 (3): 6409. doi: 10.1128/JCM.01298-15.

18.- Zhang K, Sparling J, Chow B, Elsayed S, Hussain Z, Church D. New quadriplex PCR assay for detection of methicillin and mupirocin resistance and simultaneous discrimination of Staphylococcus aureus from coagulase-negative staphylococci. J Clin Microbiol. 2004; 42 (11): 4947-55. doi: 10.1128/JCM.42.11.49474955.2004

19.- Poirel L, Walsh T, Cuvillier V, Nordmann P. (2011). Multiplex PCR for detection of acquired carbapenemase genes. Diagn Microbiol Infect Dis. 2011; 70 (1): 119-23. doi: 10.1016/j.diagmicrobio.2010.12.002.

20.- Mlynarcik P, Roderova M, Kolar M. Primer evaluation for PCR and its application for detection of carbapenemases in Enterobacteriaceae. Jundishapur J Microbiol. 2016; 9 (1): e29314. doi: 10.5812/jjm.29314.

21.- Somily A, Garaween G, Abukhalid N, Absar M, Senok A. Comparison of molecular and phenotypic methods for the detection and characterization of carbapenem resistant Enterobacteriaceae. Acta Microbiol Immunol Hung. 2016; 63 (1): 69-81. doi: 10.1556/030.63.2016.1.5.

22.- van Duin D, Doi Y. The global epidemiology of carbapenemase-producing Enterobacteriaceae. Virulence 2017; 8 (4): 460-9. doi: 10.1080/21505594.2016.1222343.

23.- Logan L, Weinstein R. The epidemiology of carbapenem-resistant Enterobacteriaceae: the impact and evolution of a global menace.
J Infect Dis. 2017; 215 (suppl 1): S28-S36. doi: 10.1093/infdis/jiw282.

24.- Lespada M, Córdova E, Roca V, Gómez N, Badia M, Rodríguez C. Bacteremia caused by Klebsiella pneumoniae carbapenemase (KPC)producing $K$. pneumoniae. A retrospective study of 7 years. Rev Esp Quimioter. 2019; 32 (1): 15-21. PMID: 30512080. https://seq.es/wpcontent/uploads/2018/12/lespada04dec2018. pdf.

25.- Cejas D, Elena A, Guevara Nunez D, Sevillano Platero P, De Paulis A, Magariños F, et al. Changing epidemiology of KPCproducing Klebsiella pneumoniae in Argentina: emergence of hypermucoviscous ST25 and high-risk clone ST307. J Glob Antimicrob Resist. 2019; 18: 238-42. doi: 10.1016/j. jgar.2019.06.005.

26.- Maya J J, Ruiz S J, Blanco V M, Gotuzzo E, Guzman-Blanco M, Labarca J, et al. Current status of carbapenemases in Latin America. Expert Rev Anti Infect Ther. 2013; 11 (7): 65767. doi: 10.1586/14787210.2013.811924.

27.- De Sousa L, Chacare M, Cuaica N, Ashby J. Primer aislamiento de Escherichia coli productora de carbapenemasa tipo New Delhi (NDM) en un hospital de Ciudad Guayana, Venezuela. A propósito de dos casos. Rev Soc Ven Microbiol. 2016; 36 (2): 40-5. http:// ve.scielo.org/pdf/rsvm/v36n2/art03.pdf

28.- Martínez D, Caña L, Rodulfo H, García J, González D, Rodríguez L, et al. Characteristics of dual carbapenemase-producing Klebsiella pneumoniae strains from an outbreak in Venezuela: a retrospective study. Rev Panam Salud Publica 2020; 44: e50. doi: 10.26633/ RPSP. 2020.50.

29.- Resurrección C, Montenegro J, Chiappe A, Vargas R, Cucho C, Mamani D, et al. Klebsiella pneumoniae Nueva Delhi metalobetalactamasa en el Hospital Nacional Dos de Mayo. Lima, Perú. Rev Per Med Exp Salud Pública 2017; 34 (2): 261-7. doi: 10.17843/ rpmesp.2017.342.2615.

30.- Silva F, Martínez O. Complejo Enterobacter cloacae. Rev Chilena Infectol. 2018; 35 (3): 297-8. doi: 10.4067/s071610182018000300297.

31.- Liu C, Qin S, Xu H, Xu L, Zhao D, Liu X, et al. New Delhi metallo- $\beta$-lactamase 1 (NDM$1)$, the dominant carbapenemase detected in carbapenem-resistant Enterobacter cloacae from Henan Province, China. PLoS One. 2015; 10 (8): e0135044. doi: 10.1371/journal. pone. 0135044.

32.- Walsh T, Weeks J, Livermore D M, Toleman M. Dissemination of NDM-1 positive bacteria in the New Delhi environment and its implications for human health: an environmental point prevalence study. Lancet Infect Dis. 2011; 11 (5): 355-62. doi: 10.1016/S14733099(11)70059-7.

33.- Yong D, Toleman M, Giske C, Cho H, Sundman K, Lee K, et al. Characterization of a new metallo- $\beta$-lactamase gene, $b l a_{\mathrm{NDM}-1}$, and a novel erythromycin esterase gene carried on a unique genetic structure in Klebsiella pneumoniae sequence type 14 from India. Antimicrob Agents Chemother. 2009; 53 (12): 5046-54. doi: 10.1128/AAC.00774-09.

34.- Narváez P, Pedroza R, Alonso G, RodríguezLemoine V. Caracterización de plásmidos de resistencia a antibióticos en aislados nosocomiales del Hospital Universitario de Caracas. Rev Soc Ven Microbiol. 2005; 25 (1): 29-34. http://ve.scielo.org/ scielo.php?script $=$ sci_arttext\&pid=S1315$25562005000100006 \& \operatorname{lng}=\mathrm{es} \& \mathrm{nrm}=\mathrm{iso} \& \operatorname{tng}=$ es.

35.- Bartolini A, Frasson I, Cavallaro A, Richter S, Palù G. Comparison of phenotypic methods for the detection of carbapenem non-susceptible Enterobacteriaceae. Gut Pathog. 2014; 613. doi: 10.1186/1757-4749-6-13.

36.- Villegas M V, Jiménez A, Esparza G, Appel T M. Carbapenemase-producing Enterobacteriaceae: A diagnostic, epidemiological and therapeutic challenge. Infectio. 2019; 23 (4): 358-68. doi: 10.22354/ in.v23i4.808. 